\title{
Editorial
}

\section{Ali Baklouti* \\ Proceedings of the 22nd Meeting of the Tunisian Mathematical Society}

https://doi.org/10.1515/apam-2018-2001

The Tunisian Mathematical Society (TMS) organizes a five-day congress each year in a different Tunisian city. The proceedings of the congress follow up on high-quality research articles published in an international peer-reviewed journal. The 22nd edition of this congress took place in Mahdia from February 25 to March 1, 2017. The present volume collects some of the important contributions to the 22nd annual meeting, entitled as above. Each paper submitted to the proceedings has been peer-reviewed through the standard process as an academic paper. The papers in this volume are all agreed upon publication with the referee, and necessary revisions, if any, have been made in the originally submitted manuscript.

This opportunity lends itself to come back on the achievements of the TMS and to make the Society better known to the general public. The TMS is a scholarly association that brings together researchers, professors and doctoral students in the field of mathematics research in Tunisia. It was founded in 1992 and it is now considered as one of the most prestigious mathematical associations in Africa. It plays a major role in stimulating and sustaining high level mathematical research in Tunisia and its surrounding region.

The role of mathematics in the human, scientific and economic development of nations is no longer to be demonstrated. It is also highly estimated that mathematical research turns out to be one of the best job providers in France as well as in several other European Union countries. The role of mathematical societies is of paramount importance in the field of education and research. The objectives of the TMS are therefore multiple, namely:

(1) To promote the discovery, learning and application of mathematics.

(2) To improve mathematics education through joint projects with mathematics teachers at all levels and in partnership with the Ministry of Higher Education and Scientific Research with support research in mathematics.

(3) To democratize mathematics by putting in place initiatives to promote and raise awareness of the discipline.

(4) To bring together Tunisian mathematicians through the publication of a monthly newsletter and the sponsorship of various activities.

(5) To develop partnerships with the Tunisian universities, companies and other mathematical associations in Tunisia and abroad.

Among the activities of the TMS that work towards the gathering of the Tunisian mathematical community, is the organization of an annual international congress where Tunisian and foreign experts are invited to give lectures in different mathematical specialties. The TMS congress is a valuable opportunity for Tunisian researchers, in Tunisia and abroad, to present their research work and interact with young doctoral students. The TMS also organizes once a year a doctoral school for students and co-organizes summer days for Tunisian mathematicians abroad in adjunction with the Mediterannean Institute of Mathematical Sciences (MIMS). It subsidizes regularly, and up to its modest budget, the holding of mathematical events throughout the country.

*Corresponding author: Ali Baklouti, Department of Mathematics, Faculty of Sciences at Sfax, Sfax University, Route de Soukra, 3000 Sfax, Tunisia, e-mail: ali.baklouti@fss.usf.tn 
The TMS is managed by an executive committee of nine elected members: a president, a vice president, a secretary general, a treasurer and five other members. It is a permanent member of the International Mathematical Union (IMU), the highest representative body of mathematics in the world.

The TMS interacts with several mathematical societies around the world and is bound by reciprocal agreements with the following companies: Swiss Mathematical Society, Mathematical Society of France, Portuguese Mathematical Society, Mathematical Society of Japan, Bulgarian Mathematical Society, Mathematical Society of Canada, Royal Spanish Mathematical Society, and Southern Africa Mathematical Sciences Association.

Can be a member of the TMS, any researcher in mathematics. Individual and institutional memberships can be made online at the TMS website (https://www.tms.rnu.tn). It has several future projects which will continue to contribute to the scientific influence of Tunisia in the world and support the elite training effort in our schools and universities.

The TMS launched recently the Tunisian Journal of Mathematics, which is an international publication organized by the society and published in electronic and print formats by the Mathematical Sciences Publishers. It publishes research articles in all areas of mathematics. These will be selected by a distinguished, international board of editors based on outstanding quality and interest, and according to the highest international standards. With the launch of this journal, the TMS contributes to the development in Africa of scientific research meeting high international standards.

Ali Baklouti, President of TMS 